\title{
Highly-magnified, multiply-imaged radio counterparts of the sub-mm starburst emission in the cluster-lens MS0451.6-0305^
}

\author{
A. Berciano Alba ${ }^{1,2}$, M. A. Garrett ${ }^{1}$, L. V. E. Koopmans ${ }^{2}$, and O. Wucknitz ${ }^{1}$ \\ 1 Joint Institute for VLBI in Europe, Postbus 2, 7990 AA, Dwingeloo, The Netherlands \\ e-mail: berciano@astro.rug.nl \\ 2 Kapteyn Astronomical Institute, University of Groningen, PO Box 800, 9700 AV, Groningen, The Netherlands
}

Received 17 March 2006 / Accepted 5 October 2006

\section{ABSTRACT}

\begin{abstract}
Context. Previous authors have reported the detection of intrinsically faint sub-mm emission lensed by the cluster MS0451.6-0305. They suggest that this emission arises from a merging system composed of a Ly-break galaxy and a pair of extremely red objects which are multiply-imaged in the optical/NIR observations.

Aims. Since the submm emission presents an unusually large angular extent $\left(\sim 1^{\prime}\right)$, the possible radio emission asociatted with that system can help to identify optical/NIR counterparts due to the higher spatial resolution and astrometric accuracy of the radio observations.

Methods. Archive VLA data (BnA configuration at $1.4 \mathrm{GHz}$ ) was reduced and analysed. A simple lens model was constructed to aid the interpretation of the radio and pre-existing sub-mm and optical/NIR data.

Results. We present a $1.4 \mathrm{GHz}$ map of the central region of MS0451.6-0305 and report the detection of gravitationally lensed radio emission, coincident with the previously discovered sub-mm lensed emission. The overall morphology and scale of the radio and sub-mm emission are strikingly similar, extending $\sim 1^{\prime}$ across the sky. This observation strongly suggests that the radio and sub-mm emission arise from the same sources. Preliminary estimates of the total $S_{850 \mu \mathrm{m}} / S_{1.4 \mathrm{GHz}}$ flux density ratio appear to be consistent with that expected from distant star forming galaxies. The radio emission is resolved into 7 distinct components, and the overall structure can be explained, using a simple lens model, with three multiply-imaged radio sources at $z \sim 2.9$. One of these sources is predicted to lie in the middle of the previously mentioned merging system in the source plane, suggesting that it is related to the intense star formation generated during the merging process.
\end{abstract}

Key words. gravitational lensing - galaxies: starburst - radio continuum: galaxies - galaxies: clusters: individual: MS0451.6-0305

\section{Introduction}

Sub-mm galaxies (SMGs) were first detected by SCUBA ${ }^{1}$ (Smail et al. 1997) and are believed to be dusty star forming galaxies located at high redshift (Ivison et al. 2002; Smail et al. 2002; Chapman et al. 2003). It is also suggested that they are the progenitors of present-day massive elliptical galaxies (e.g. Lilly et al. 1999; Swinbank et al. 2006). Little is known about the objects associated with the faint end of the SMG population $\left(S_{850 \mu \mathrm{m}}<2 \mathrm{mJy}\right)$, but they are predicted to dominate (energetically) the population as a whole (Knudsen 2004). A recent statistical stacking analysis (Knudsen et al. 2005), suggests that distant red galaxies (DRGs) and Extremely Red Objects (EROs) contribute $\sim 50 \%$ of the flux density of sub-mm sources with $0.5<S_{850 \mu \mathrm{m}}<5 \mathrm{mJy}$.

Intrinsically faint SMG cannot easily be detected, since their flux densities lie below the $\sim 2$ mJy confusion limit of SCUBA images at $850 \mu \mathrm{m}$. Typically they also fall well below the (thermally limited) sensitivity of current radio instruments, such as the VLA. Individual systems can often only be detected via strong gravitational lensing effects, produced by massive

\footnotetext{
* Appendix tables are only available in electronic form at http://www . aanda.org

1 Submillimetre Common-User Bolometer Array, mounted at the James Clerk Maxwell Telescope (JCMT).
}

foreground clusters of galaxies (Knudsen 2004; Kneib et al. 2004; Garrett et al. 2005).

The spatial magnification provided by the lensing cluster overcomes instrumental confusion limitations in the sub-mm and also boosts the measured flux density of the source (provided the lensed images remain unresolved), thereby increasing the probability of detection (Blain 1997). Another advantage of cluster lensing is that the magnification provided by the lens effectively increases the spatial resolution of the observations, with the largest magnifications usually occurring in cases of multiple imaging.

SMM J16359+6612, associated with the cluster Abell 2218, was the first intrinsically faint, multiply imaged SMG detected in both the sub-mm (Kneib et al. 2004) and radio (Garrett et al. 2005). In this paper, we present VLA $1.4 \mathrm{GHz}$ radio observations of a second case, SMM J04542-0301 (Chapman et al. 2002a), associated with the cluster MS0451.6-0305.

MS0451.6-0305 is a cluster of galaxies situated at $z=0.55$ (Gioia \& Luppino 1994) that has recently been studied using optical and near-Infrared (NIR) data (Borys et al. 2004). They conclude that the sub-mm emission is probably related to an interacting system of three objects lying at $z \sim 2.9$ : a Lyman Break Galaxy (LBG) and a pair of Extremely Red Objects (EROs). In the optical and NIR images, it is proposed that the LBG is imaged into two visible arcs, and the ERO pair are responsible for 


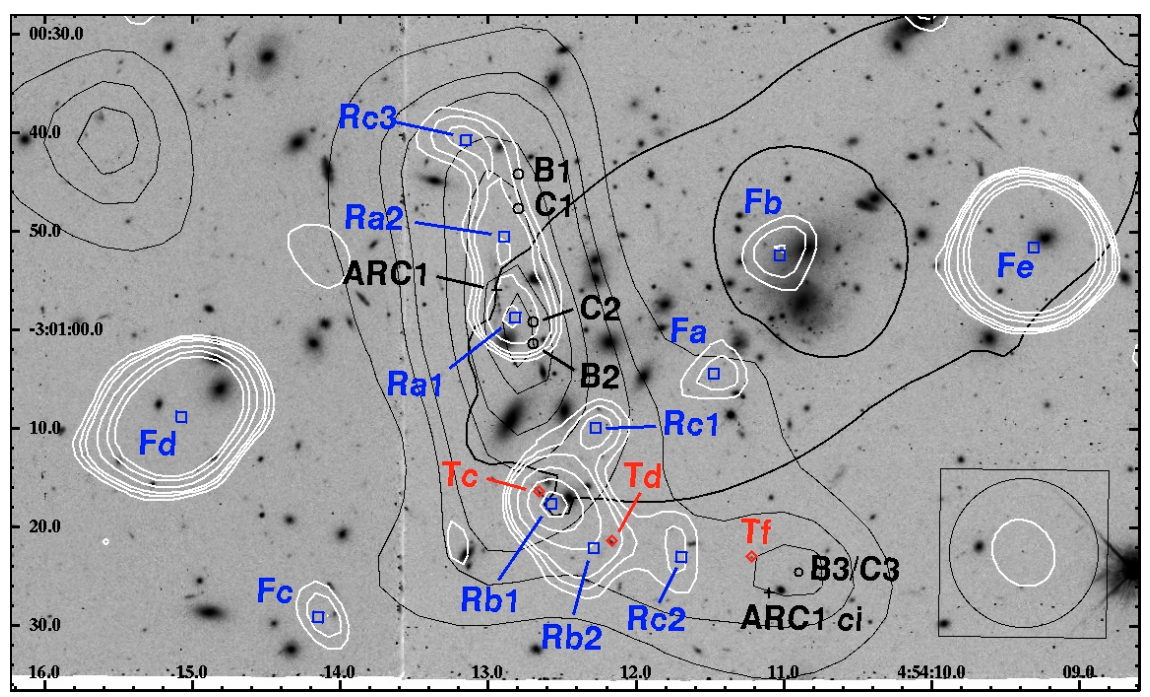

Fig. 1. The VLA 1.4 GHz naturally weighted contour map (solid white lines) superimposed upon the SCUBA 850- $\mu$ m contour map (solid thin black lines) and the inverted HST F702W image of the centre of the cluster MS0451.6-0305 (Borys et al. 2004). The axes represent the right ascension ( $x$-axis) and declination ( $y$-axis) in the J2000 coordinate system. The solid thick black curves are the tangential (outer) and radial (inner) critical lines at $z=2.911$ associated with the lens model of the cluster determined by Borys et al. (2004). The boxes are the positions obtained via Gaussian fits of the radio sources. The diamonds are the positions of three EROs from Takata et al. (2003), and the crosses/circles are the positions of a LBG lensed as two arc (ARC1 and ARC1 ci), and a triply-imaged EROs pair: B1/B2/B3 (images of ERO B) and C1/C2/C3 (images of ERO C) (see Borys et al. 2004). The squares and circles have a size of $1^{\prime \prime}$ to illustrate the random and systematic errors due to measurement indeterminations and the aligment of the different images. Contours of the radio map are drawn at $-3,3,4,5,8,12$ and 16 times the $1-\sigma$ noise level of $9 \mu \mathrm{Jy}$ per beam. Contours of the sub-mm map are drawn at 4, 6, 7, 9, 10, 11 and $11.5 \mathrm{mJy}$ per beam. The white circle inside a box in the bottom-right corner is the beam-size of the radio map $\left(6.99 \times 6.03\right.$ arcsec in position angle $\left.\mathrm{PA}=32.6^{\circ}\right)$ whereas the black one corresponds to the beam-size of the sub-mm map $(15 \times 15$ arcsec $)$.

5 additional sources of emission in the field (Borys et al. 2004; Takata et al. 2003). However, the emission coming from the north-eastern and the central regions of the sub-mm image, cannot be fully reproduced using the LBG and the ERO pair alone (see Fig. 7 from Borys et al. 2004).

In this paper, we present deep, high resolution $1.4 \mathrm{GHz}$ VLA observations of SMM J04542-0301. In Sect. 2, we describe the VLA data analysis and present the associated radio images. Section 3 describes a simple lens model for the system, in an attempt to explain the lensed nature of the radio emission related to SMM J04542-0301. In Sect. 4, we compare the radio and sub-mm emission, including a discussion about possible optical/NIR counterparts, and the preliminary calculation of the $S_{850 \mu \mathrm{m}} / S_{1.4 \mathrm{GHz}}$ flux density ratio. A summary of our main results is presented in Sect. 5. In the following discussion, we assume a $\Lambda \mathrm{CDM}$ cosmological model with $\Omega_{m}=0.3, \Omega_{\lambda}=0.7$ and $h_{0}=0.7$.

\section{Radio observations}

VLA 1.4 GHz observations of the cluster MS0451.6-0305 were made in June 2002, and were retrieved from the NRAO data archive system ${ }^{2}$. The integration time was $7.8 \mathrm{~h}$ with the VLA in BnA configuration, employing two $25 \mathrm{MHz}$ IFs in both left and right-hand circular polarization. Each IF was subdivided into 7 channels. The data analysis was performed using the NRAO AIPS package using standard analysis techniques. The absolute flux density scale was set by observations of $0137+331$, and phase calibration was performed via short observations of $0503+020$ between the $1 \mathrm{~h}$ target scans. A wide-field image was made and bright sources far from the field centre were subtracted

\footnotetext{
${ }^{2}$ Project ID AN0109, PI: Nakanishi.
}

from the data. Self-calibration using the remaining sources in the centre of the field realised images with a $1 \sigma$ rms noise level of $9 \mu \mathrm{Jy} /$ beam.

In Fig. 1, we present the radio contour map (solid white lines) of the naturally weighted VLA image of SMM J04542-0301, superimposed upon the HST F702W image and the sub-mm contour map (solid thin black lines) presented in Borys et al. (2004). Note that the SCUBA beam is $15 \times 15$ arcsec, significantly larger than the VLA beam $(6.99 \times$ $6.03 \mathrm{arcsec}$ ). To compare the radio and sub-mm emission at the same resolution (see Sect. 4), we also produced a tapered image of the radio data, weighting down the long baselines to reach a Gaussian restoring beam similar to the SCUBA beam. The resulting map is presented in Fig. 3 (solid white lines).

The AIPS task IMFIT was used to fit Gaussian components to all the radio sources detected in the field ( 3 and 4 Gaussians simultaneously, in the case of the two extended regions of radio emission in the naturally weighted map). The radio positions obtained are represented by square boxes in Fig. 1. The results are listed in Table 1, together with their formal errors.

Radio source components $\mathrm{Ra}, \mathrm{Rb}, \mathrm{Rc}$ and $\mathrm{Fa}$ appear to be related to the sub-mm emission. The source $\mathrm{Fb}$ may be related to the central brightest cluster galaxy (BCG) (see Table 6), but there is no obvious optical/NIR counterpart for Fc.

We also detect two bright radio sources, Fd and Fe. The latter is clearly identified with a optical/NIR counterpart, and both radio sources are almost two orders of magnitude brighter than the other radio sources in the field. These sources are probably not lensed images of the same background source - Fe is more compact than Fd, even though it is brighter. The positions of $\mathrm{Fd}$ and $\mathrm{Fe}$ are coincident (within the errors) with two radio sources already reported in Stocke et al. (1999) (see Table 6). 
Table 1. Details of the radio sources observed in the core of MS0451.6-0305. The columns show: position (RA, Dec), peak flux density ( $S_{\mathrm{Pk}}$ ), total flux density $\left(S_{\mathrm{T}}\right)$ and deconvolved Gaussian sizes (major axis, minor axis and position angle) with their corresponding formal errors. Cases where the parameters of the Gaussian fits are not well constrained are indicated by a dash. The coordinates are given as offsets with respect to the cluster centre, $\mathrm{RA}(\mathrm{J} 2000)=04: 54: 10.8$ and $\operatorname{Dec}(\mathrm{J} 2000)=-03: 00: 51.6$ (see Takata et al. 2003, Table 2). A version of this table in absolute coordinates can be found in the online material.

\begin{tabular}{lccccccc}
\hline \hline Name & $\begin{array}{c}\text { RA } \\
\text { J2000 (") }\end{array}$ & $\begin{array}{c}\text { Dec } \\
\text { J2000 }\left(^{(\prime)}\right)\end{array}$ & $\begin{array}{c}S_{\mathrm{Pk}} \\
\mu \mathrm{Jy}\end{array}$ & $\begin{array}{c}S_{\mathrm{T}} \\
\mu \mathrm{Jy}\end{array}$ & $\begin{array}{c}\text { Maj Axis } \\
\left({ }^{\prime \prime}\right)\end{array}$ & $\begin{array}{c}\text { Min Axis } \\
\left({ }^{\prime \prime}\right)\end{array}$ & $\begin{array}{c}\text { PA } \\
\mathrm{deg}\end{array}$ \\
\hline $\mathrm{Ra} 2$ & $31.3 \pm 0.3$ & $1.1 \pm 0.4$ & $70 \pm 8$ & $95 \pm 18$ & $6 \pm 1$ & $2 \pm 1$ & $27 \pm 28$ \\
$\mathrm{Ra} 1$ & $30.3 \pm 0.2$ & $-7.1 \pm 0.2$ & $109 \pm 9$ & $109 \pm 9$ & - & - & - \\
$\mathrm{Rb} 1$ & $26.5 \pm 0.2$ & $-26 \pm 0.2$ & $151 \pm 9$ & $151 \pm 9$ & - & - & - \\
$\mathrm{Rb} 2$ & $22.3 \pm 0.5$ & $-30.50 \pm 0.7$ & $52 \pm 8$ & $100 \pm 22$ & $9 \pm 2$ & $3 \pm 2$ & $158 \pm 14$ \\
$\mathrm{Rc} 1$ & $22.1 \pm 0.6$ & $-18.3 \pm 0.4$ & $50 \pm 9$ & $55 \pm 16$ & $6 \pm 2$ & - & $112 \pm 11$ \\
$\mathrm{Rc} 2$ & $13.5 \pm 0.6$ & $-31.3 \pm 0.8$ & $41 \pm 8$ & $58 \pm 18$ & $7 \pm 2$ & $1 \pm 3$ & $10 \pm 163$ \\
$\mathrm{Rc} 3$ & $35.4 \pm 0.8$ & $10.9 \pm 0.5$ & $52 \pm 8$ & $78 \pm 19$ & $8 \pm 2$ & $0 \pm 2$ & $73 \pm 11$ \\
\hline $\mathrm{Fa}$ & $10.1 \pm 0.6$ & $-12.7 \pm 0.6$ & $45 \pm 9$ & $50 \pm 17$ & $4 \pm 6$ & $0 \pm 4$ & $123 \pm 38$ \\
$\mathrm{Fb}$ & $3.5 \pm 0.6$ & $-0.8 \pm 0.6$ & $49 \pm 9$ & $70 \pm 20$ & $6 \pm 5$ & $3 \pm 6$ & $122 \pm 45$ \\
$\mathrm{Fc}$ & $50.4 \pm 0.6$ & $-37.5 \pm 0.6$ & $44 \pm 9$ & $44 \pm 9$ & - & - & - \\
$\mathrm{Fd}$ & $64.1 \pm 0.1$ & $-17.2 \pm 0.1$ & $634 \pm 9$ & $1039 \pm 21$ & $7.6 \pm 0.1$ & $1.4 \pm 0.5$ & $128 \pm 1$ \\
$\mathrm{Fe}$ & $-22.40 \pm 0.02$ & $-0.07 \pm 0.02$ & $1549 \pm 9$ & $1777 \pm 17$ & $3.3 \pm 0.1$ & $0.8 \pm 0.5$ & $125 \pm 3$ \\
\hline
\end{tabular}

\section{A lens model of the radio emission}

In order to aid our interpretation of the radio emission associated with SMM J04542-0301, we have created a simple elliptical lens model with external shear to describe the cluster lens potential. Our analysis employs the GRAVLENS software package developed by Keeton (2001). The modeling strategy is described in Sect. 3.1, and the results of the model are discussed in Sect. 3.2.

\subsection{Modeling strategy}

The first lens model of MS0451.6-0305 was presented in Takata et al. (2003), and describes the total mass distribution of the cluster by a singular isothermal ellipsoid. To be more sensitive to the local mass distribution, Borys et al. (2004) modeled the cluster core and 39 galaxy cluster members using 40 smoothly truncated pseudo-isothermal elliptical mass distribution profiles (PIEMD, see Kneib et al. 1996).

The critical curves of the best lens model found by Borys et al. (2004) are shown in Fig. 1. It can be seen that the tangential critical curve lies between the radio emission Ra1-Ra2, $\mathrm{Rb} 1-\mathrm{Rb} 2$ and Rc1-Rc2. Based on the general properties of the lens geometry, this suggests that each of these image pairs belongs to a group of 3 images produced by one source located close to the caustic in the source plane. We propose the following scenario in an attempt to understand the radio emission we observe in terms of gravitational lensing:

1. Ra2-Ra1 are fold images of a source Ra, with an expected counterpart image ( $\mathrm{Ra} 3$ ) close to ARC1ci.

2. $\mathrm{Rb} 1-\mathrm{Rb} 2$ are fold images of a source $\mathrm{Rb}$ with an expected counterpart image (Rb3) close to Rc3.

3. Rc1-Rc2-Rc3 are multiple images of a single source Rc, located behind the cluster.

To test this hypothesis, we implemented a new lens model for MS0451.6-0305 using the GRAVLENS code. Since we are only interested in testing the lensed nature of the radio emission, we modeled the overall mass distribution of the cluster using a single mass profile plus external shear (see Appendix A for a more detailed description of the related formulae). Unlike the two previous models, we choose an NFW profile (Navarro et al. 1996) for the cluster mass distribution that is consistent with observations (see e.g. Pointecouteau et al. 2005; Comerford et al. 2006;
Table 2. Optical/NIR constraints used in the lens model. The columns show: position (RA, Dec), total flux density in $K^{\prime}$ band and predicted magnification $\mu$ (see also Table 1 in B04). The coordinates are given as offsets with respect to the cluster centre, RA(J2000) $=04: 54: 10.8$ and $\operatorname{Dec}(\mathrm{J} 2000)=-03: 00: 51.6$ (see Takata et al. 2003, Table 2).

\begin{tabular}{lcccc}
\hline \hline Name & $\begin{array}{c}\text { RA } \\
\text { J2000 (") }\end{array}$ & $\begin{array}{c}\text { Dec } \\
\text { J2000 }\left({ }^{(\prime)}\right)\end{array}$ & $\begin{array}{c}\text { Flux in } K^{\prime} \text { band } \\
\mu \mathrm{Jy}\end{array}$ & $\mu$ \\
\hline B1 & 29.95 & 7.498 & $3.6 \pm 0.1$ & $8 \pm 1$ \\
B2 & 28.47 & -9.702 & $1.9 \pm 0.1$ & $10 \pm 1$ \\
C1 & 29.95 & 4 & $1.4 \pm 0.1$ & $10 \pm 1$ \\
C2 & 28.47 & -7.502 & $0.9 \pm 0.1$ & $5 \pm 1$ \\
B3/C3 & 1.481 & -32.9 & $2.5 \pm 0.1$ & $5 \pm 1$ \\
\hline
\end{tabular}

Bassino et al. 2006) and predictions from dark matter simulations.

To constrain the model, we performed a number of distinct steps. First, the parameters of the mass model were chosen in order to reproduce the general shape of the critical lines determined by Borys et al. (2004) (see Fig. 1). Second, the positions and fluxes of the ERO images (B1, C1, B2, C2, B3/C3) were used as constraints for the first optimisation of the input model. And third, we included the positions and fluxes of the radio images as new constraints (following the previous hypothesis) to re-optimise the model. The constraints are listed in Tables 1 and 2 . The coordinates of the cluster centre used in Takata et al. (2003) were chosen as the origin of the coordinate system.

The lens model obtained through this process turned out to have a degeneracy between the mass and the scale radius. To break this degeneracy, we used information about the concentration parameter $\left(\delta_{\mathrm{c}}\right)$ derived from $\Lambda \mathrm{CDM} N$-body simulations. First, we produced a set of 10 new models varying the core radius between 40 and 150 (a range that contains the core radius value of the degenerate model). Following the formalism presented in Bullock et al. (2001), we calculated the concentration parameter and the virial mass for the set of new models (see Appendix A for details). We found that, while $\delta_{\mathrm{c}}$ varies between 3.3 and 9.6, the virial mass is always of the order of $10^{15} M_{\odot}$. For a halo of that mass situated at $z=0.55$, the toy model presented in Bullock et al. (2001) predicts a concentration parameter of $\delta_{\mathrm{c}}=3.35$. So we plotted $\delta_{\mathrm{c}}$ versus the core radius for the set of new models, interpolating the results in order to determine the core radius that corresponds to $\delta_{\mathrm{c}}=3.35$. 

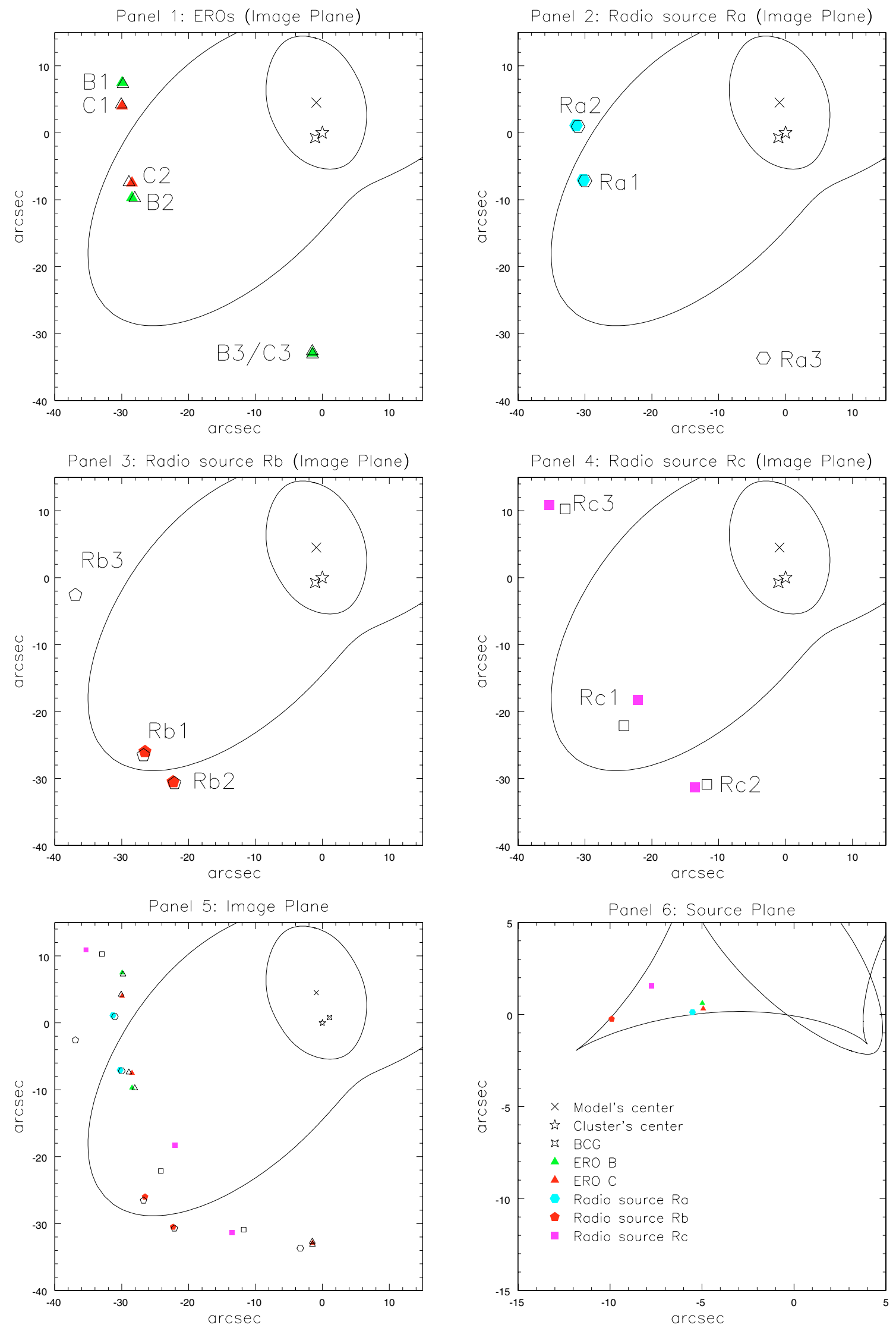

Fig. 2. Lens model results. The solid black curves represent the critical curves (panels 1-5) and caustics (panel 6) that define the model. Filled symbols represent the measured positions of the ERO images (Borys et al. 2004) and the radio components; empty symbols are the positions predicted by the model. The ERO pair and each group of suggested multiply-imaged radio components are shown seperately in different panels (1 to 4). Panel 5 shows all of these components plotted together. Panel 6 shows the position of the ERO pair and radio sources in the source plane. 
Finally, we fixed the core radius of the model to this value and re-optimized the remaining parameters.

\subsection{Results}

Figure 2 and Table 3 show the results of the lens model. Although the model is not unique, it is able to reproduce the positions of the ERO images and the radio emission (Ra1, Ra2, $\mathrm{Rb} 1$, and Rb2) very well. The largest offsets are found for Rc1, Rc2 and Rc3 (see panel 4 in Fig. 2) which are the most distant images from the critical curves. This is consistent with the effect of degeneracies in the global mass model near the critical curves: a change in the model parameters produces a more significant change in the image properties when they are located further from the critical curves.

To improve the fit of images Rc1-Rc2-Rc3, the redshift of the model was changed for source Rc, but this only produces a radial shift of their predicted positions in the same direction, something that cannot improve the fit shown in panel 4. Therefore, we believe that the most important contribution to this offset is probably coming from the group of galaxies in the region between Ra1 and Rc1 (see Fig. 1). This group of galaxies is expected to introduce perturbations in the overall mass distribution of the cluster which are not accounted for in the smooth NFW mass model.

One notable result is that the model predicts two faint counterpart images ( $\mathrm{Ra} 3$ and $\mathrm{Rb} 3$ ) that do not appear in our radio image. In our model Ra3 and Rb3 are less magnified than Ra1-Ra2 and $\mathrm{Rb} 1-\mathrm{Rb} 2$ respectively. The predicted relative magnifications suggest that Ra3 and Rb3 should appear in our maps at the 2 and $4 \sigma$ level respectively, but there is no evidence for this in the radio images. We note, however, that the predicted magnifications depend strongly on the overall mass model employed and perturbations by individual galaxies, so they should be treated as rough estimates of the true magnification. Therefore, the nondetection of these images do not necessarily mean that the lens model is wrong, since the real magnification could be less than that predicted by this simple model.

The mass model parameters that characterise the NFW profile are summarised in Table 4. The errors represent the $1 \sigma$ level of the $\chi^{2}$ function of each parameter. We note that the shear of the model is quite large, and the model centre is shifted $\Delta \mathrm{RA}=-0.9^{\prime \prime}$ and $\Delta \mathrm{Dec}=4.5^{\prime \prime}$ from the assumed position of the cluster centre. These effects are most likely systematic errors that compensate for the contribution of the group of galaxies that we are not including in the model, and the fact that we are forcing it to fit $\mathrm{Rb} 1$ and $\mathrm{Rb} 2$ as mirror images (a hypothesis that we will discuss in detail in Sect. 4).

As already noted, this lens model is only meant to test if the configuration of the observed radio emission can be understood as the result of gravitational lensing. In that sense, Fig. 2 shows that the model is able to reproduce the position of the EROs and the radio observations reasonably well, explaining the morphology of the radio map as the result of three lensed background radio sources. Therefore, we expect the two EROs and the three radio sources lie at the same redshift in the source plane $(z \sim 2.9$, estimated in Borys et al. (2004) for the EROs including the spectroscopic redshift information of the ARC in their lens model).

But perhaps the most interesting result from the lens model is that the source $\mathrm{Ra}$ and the EROs B and $\mathrm{C}$ are predicted to be located inside a region of about 0.6 arcsec in the source plane (see panel 6 of Fig. 2), which corresponds to a linear separation of only $4.7 \mathrm{kpc}$. The same situation is found in the case of the LBG and the ERO pair, which are separated by $\sim 10 \mathrm{kpc}$ in the source plane (see Borys et al. 2004). This means that the radio source Ra is lying just between the LBG and the EROs, all of which are located in a region smaller than the extent of a typical galaxy ( $\sim 20 \mathrm{kpc})$. It would appear therefore, that the radio source $\mathrm{Ra}$, the LBG and the ERO pair indeed constitute an interacting or merging system of galaxies.

\section{Comparison of the radio, sub-mm and optical/NIR data}

In this section we make a more detailed comparison between our radio maps and the pre-existing sub-mm and NIR/optical data (Borys et al. 2004; Takata et al. 2003).

\subsection{Sub-mm vs. Radio emission: morphology and flux density ratio}

Figure 3 presents the sub-mm and tapered radio maps with a common resolution of $\sim 15^{\prime \prime} \times 15^{\prime \prime}$. The regions of radio and sub-mm emission are not only coincident, but they are extended on the same angular scale $\left(\sim 1^{\prime}\right)$ and have a strikingly similar morphology. This strongly suggests that the radio and sub-mm emission are associated with each other and are produced by the same (lensed) sources, probably star forming galaxies at $z>2$. In addition, the positions of the radio components located at $\mathrm{Rb} 1$, $\mathrm{Rb} 2, \mathrm{Rc} 1, \mathrm{Rc} 2$ and Fa (see Fig. 1) are consistent with the sub$\mathrm{mm}$ emission that could not be reproduced using only the ERO images and ARC1 (see Borys et al. 2004, Fig. 7).

However, Fig. 3 also shows some differences in the morphology of the radio and sub-mm emission. The most relevant one is that the brightest region in sub-mm is not associated with the brightest region in radio $(\mathrm{Rb} 1 / \mathrm{Rb} 2)$, but with the second brightest (Ra1/Ra2). One explanation for this apparent discrepancy in $\mathrm{Rb} 1 / \mathrm{Rb} 2$, is that the radio and sub-mm emission arise from slightly different regions in the source plane and are differentially magnified. This effect could be quite significant for sources lying close to or extending across a caustic. Indeed, we note that recent Mid-IR and radio studies of local star forming galaxies show variations across the disk of up to a factor of $\sim 5$ in the ratio of the FIR and radio luminosity (Murphy et al. 2004). Another possibility is that the radio emission in the region $\mathrm{Rb} 1 / \mathrm{Rb} 2$ is not only associated with the sub-mm emission that arises from high- $z$ star formation but from an additional component, perhaps an AGN in the foreground cluster that has no counterpart in the sub-mm. Indeed, a possible galaxy cluster member is located within $1.5^{\prime \prime}$ of the radio component $\mathrm{Rb} 1$, and may be an optical/NIR counterpart to this source (see Table 6).

The tapered map (see Fig. 3) also shows an extension of the radio emission towards B3/C3 which is not seen in Fig. 1. This suggests the possible existence of an extended radio source in this region, presumably associated with the faint sub-mm emission "toe" that appears in the image presented by Borys et al. (2004). This is consistent with the existence of the radio counterpart Rc3 predicted by the lens model.

Assuming that the radio and sub-mm emission is produced by the same galaxies, the $S_{850 \mu \mathrm{m}} / S_{1.4 \mathrm{GHz}}$ flux density ratio provides information about their SEDs. Note that, since the sub-mm image does not resolve the radio components shown in Fig. 1 due to the poor resolution of SCUBA, the radio flux density should be obtained from the tapered radio map. The integrated flux densities were calculated with the AIPS task TVSTAT, using the $4 \mathrm{mJy} /$ beam sub-mm contour to delimit the same integration area in the sub-mm and tapered radio maps (see Fig. 3). Using this method, we find $S_{850 \mu \mathrm{m}}=54.6 \pm 5.7 \mathrm{mJy}$ and 
Table 3. Lens model results. The columns show: coordinates of the predicted images (RA, Dec), predicted lensed flux $\left(S_{\mathrm{T}}\right)$, offsets between the measured and predicted quantities $\left(\Delta \mathrm{RA}, \Delta \mathrm{Dec}, \Delta S_{\mathrm{T}}\right)$ and predicted magnification $(\mu)$. The coordinates are given as offsets with respect to the cluster centre, $\mathrm{RA}(\mathrm{J} 2000)=$ 04:54:10.8 and $\operatorname{Dec}(\mathrm{J} 2000)=-03: 00: 51.6$ (see Takata et al. 2003, Table 2).

\begin{tabular}{lccccccc}
\hline \hline Name & $\begin{array}{c}\text { RA } \\
\left({ }^{\prime \prime}\right)\end{array}$ & $\begin{array}{c}\Delta \mathrm{RA} \\
\left({ }^{\prime \prime}\right)\end{array}$ & $\begin{array}{c}\mathrm{Dec} \\
\left({ }^{\prime \prime}\right)\end{array}$ & $\begin{array}{c}\Delta \mathrm{Dec} \\
\left({ }^{\prime \prime}\right)\end{array}$ & $\begin{array}{c}S_{\mathrm{T}} \\
\mu \mathrm{Jy}\end{array}$ & $\begin{array}{c}\Delta S_{\mathrm{T}} \\
\mu \mathrm{Jy}\end{array}$ & $\mu$ \\
\hline $\mathrm{B} 1$ & -29.80 & -0.15 & 7.25 & -2.75 & 2.82 & 0.75 & 12.19 \\
$\mathrm{~B} 2$ & -28.05 & -0.42 & -9.76 & 0.06 & 2.70 & 0.83 & -11.66 \\
$\mathrm{~B} 3$ & -1.50 & 0.02 & -32.66 & -0.24 & 1.13 & 0.11 & 4.89 \\
$\mathrm{C} 1$ & -30.07 & 0.12 & 4.23 & -0.23 & 1.31 & 0.11 & 17.66 \\
$\mathrm{C} 2$ & -28.92 & 0.45 & -7.40 & -0.1 & 1.26 & 0.36 & -17.00 \\
$\mathrm{C} 3$ & -1.47 & -0.01 & -33.14 & 0.24 & 0.35 & 0.88 & 4.75 \\
\hline $\mathrm{R} 2$ & -31.03 & -0.29 & 0.94 & 0.17 & 100.95 & -5.95 & 25.18 \\
$\mathrm{Ra} 1$ & -29.99 & -0.26 & -7.18 & 0.12 & 99.23 & -9.77 & -24.76 \\
$\mathrm{R} 3$ & -3.30 & - & -33.67 & - & 19.73 & - & 4.92 \\
$\mathrm{Rb} 1$ & -26.75 & 0.23 & -26.52 & 0.54 & 131.12 & -19.88 & -36.78 \\
$\mathrm{Rb} 2$ & -22.11 & -0.22 & -30.70 & 0.2 & 111.05 & -11.05 & 31.15 \\
$\mathrm{Rb3}$ & -36.93 & - & -2.58 & - & 37.65 & - & 10.56 \\
$\mathrm{Rc} 1$ & -24.16 & 2.11 & -22.12 & 3.86 & 72.17 & 17.17 & -11.51 \\
$\mathrm{Rc} 2$ & -11.76 & -1.76 & -30.90 & -0.43 & 62.34 & -4.34 & 9.94 \\
$\mathrm{Rc} 3$ & -32.96 & -2.39 & 10.28 & 0.63 & 42.37 & 35.63 & 6.75 \\
\hline
\end{tabular}

Table 4. Lens model parameters. The columns show: mass scale $\left(\kappa_{\mathrm{s}}\right)$, galaxy position in $\operatorname{arcsec}\left(x_{0}, y_{0}\right)$, ellipticity $\left(e, \theta_{\mathrm{e}}\right)$, external shear $\left(\gamma, \theta_{\gamma}\right)$ and scale radius in $\operatorname{arcsec}\left(r_{\mathrm{s}}\right)$. The errors represent the 1- $\sigma$ level of the $\chi^{2}$ fuction for each parameter. The scale radius has no error estimations because its value was fixed during the optimization process to be consistent with a concentration parameter of 3.35 (see Sect. 3.1).

\begin{tabular}{lcccccccc}
\hline \hline Mass Model & $\kappa_{\mathrm{s}}$ & $x_{0}$ & $y_{0}$ & $e$ & $\theta_{\mathrm{e}}$ & $\gamma$ & $\theta_{\gamma}$ & $r_{\mathrm{s}}$ \\
\hline \multirow{2}{*}{$\mathrm{NFW}$} & $0.33_{-0.01}^{+0.01}$ & $-0.90_{-1.2}^{+0.7}$ & $4.51_{-1.4}^{+0.9}$ & $0.55_{-0.03}^{+0.03}$ & $-42.20_{-5.6}^{+1.1}$ & $0.18_{-0.02}^{+0.02}$ & $61.44_{-8.4}^{+2.4}$ & {$[142.90]$} \\
\hline
\end{tabular}

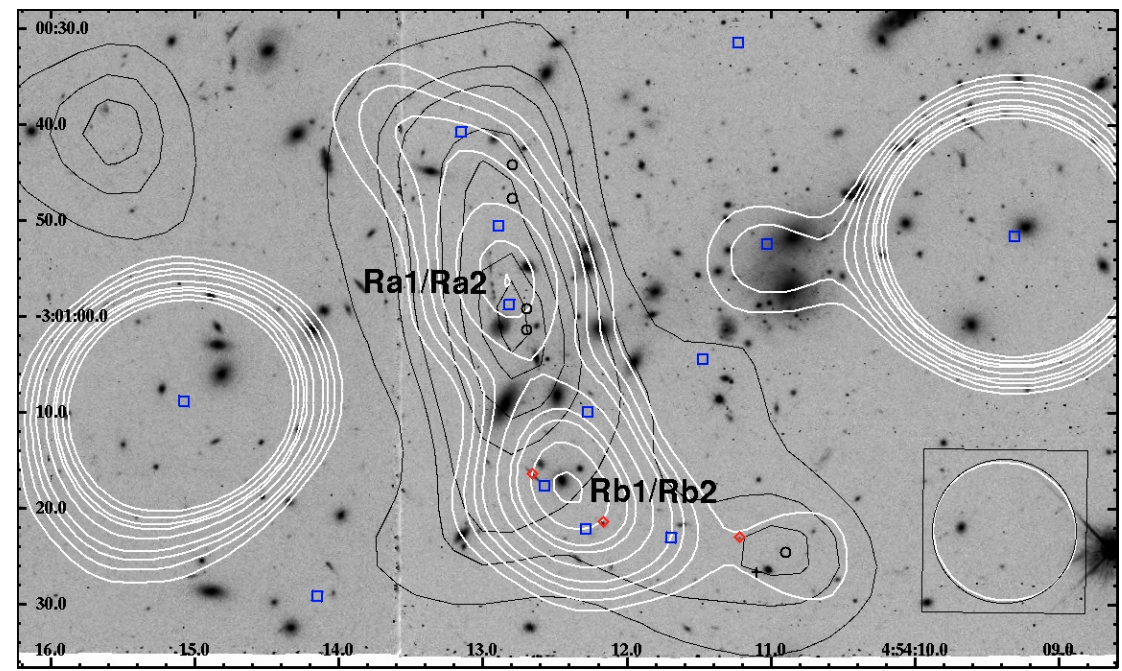

Fig. 3. The VLA $1.36 \mathrm{GHz}$ tapered contour map (solid white lines) superimposed upon the SCUBA 850- $\mu$ m contour map (solid black lines) and the inverted HST F702W image of the centre of the cluster MS0451.6-0305 (Borys et al. 2004). The axes represent the right ascension ( $x$-axis) and declination ( $y$-axis) in the $\mathbf{J} 2000$ coordinate system. Contours of the tapered radio map are drawn at $-3,3,4,5,6,8,10,11,13$ and 15 times the 1- $\sigma$ noise level of $14.2 \mu \mathrm{Jy} / \mathrm{beam}$. Contours of the sub-mm map are drawn at 4, 6, 7, 9, 10, 11 and $11.5 \mathrm{mJy} / \mathrm{beam}$. The positions of the radio sources and relevant objects in the NIR (see Fig. 1), are plotted as reference points. The black circle inside the box in the bottom-right corner is the beam size of the sub-mm map $(15 \times 15$ arcsec), whereas the white one (almost covered by the black circle) corresponds to the beam-size of the radio map $\left(15.06 \times 14.26\right.$ arcsec, in position angle $\left.\mathrm{PA}=68.3^{\circ}\right)$.

$S_{1.4 \mathrm{GHz}}=0.547 \pm 0.03 \mathrm{mJy}$. The errors were calculated using the expression $\sigma_{\text {beam }} \times \sqrt{N}$, where $\sigma_{\text {beam }}$ is the noise per beam of the image, and $N$ is the number of beams within the area delimited by the $4 \mathrm{mJy} /$ beam sub-mm contour.

The observed $S_{850 \mu \mathrm{m}} / S_{1.4 \mathrm{GHz}}$ flux density ratio in MS0451.6-0305 was compared with the flux density ratio obtained from a set of SED galaxy templates. This set is composed by the archetype star forming galaxies Arp 220 and M 82 (Polletta et al. in prep.), the AGN-dominated galaxy Mrk 231, and the set of Blue Compact Dwarf galaxy SEDs presented in Hunt \& Maiolino (2005). To detect possible differences in the nature of faint and bright sub-mm sources, we also performed the same analysis for the faint sub-mm source detected in A2218, and the bright source detected in A1835 (see Table 5).

We find that the observed flux density ratio in MS0451.6 0305 is closer to the one obtained using the SED template of M 82. However, we note that the observed total flux ratio is being underestimated due to the "excess" of radio emission 
Table 5. Flux density ratios. The columns show: name of the sub-mm emission (SMM), redshift of the sub-mm source (z), cluster in which the sub-mm emision is located (Cluster lens), SED template that provides the best fit to the observed flux ratio (Best SED template), flux density ratio obtained from observations (FDR observed), flux densiy ratio predicted by the template (FDR template), deviation between the observed and predicted flux ratio (deviation). The value of FDR observed in SMM J16359+6612 was calculated by adding the fluxes of all the images together. The deviations were calculated as (FDR template/FDR observed) -1 .

\begin{tabular}{cccccccc}
\hline \hline SMM & $z$ & Cluster lens & Best SED template & FDR observed & FDR template & deviation & References \\
\hline J14011+0252 & 2.56 & A1835 & NGC 5253 & $127 \pm 37$ & 115 & -0.09 & I01 \\
J16359+6612 & 2.516 & A2218 & Mrk 231 & $60 \pm 10$ & 49 & -0.18 & K04; G05 \\
J04542-0301 & 2.9 & MS0451.6-0305 & M 82 & $100 \pm 11$ & 115 & 0.15 & Sect. 4.1 \\
\hline
\end{tabular}

associated with $\mathrm{Rb} 1 / \mathrm{Rb} 2$, and therefore a SED similar to Arp 220 may be more appropriate for this source. Until the sub$\mathrm{mm}$ emission can be resolved into different components to determine their flux ratios independently, all we can conclude is that the overall flux density ratio is largely consistent with the favored hypothesis that the bulk of the radio/sub-mm emission is arising from distant star forming galaxies that appear to follow the well known FIR-radio correlation (Condon 1992; Garrett 2002).

The bright source in A1835 is the only one which is well fitted by one of the BCD templates presented by Hunt \& Maiolino (2005). The faint source in A2218 seems to be similar to Mrk 231, perhaps suggesting it is a "warm" SCUBA source following the classification presented in Egami et al. (2004).

\subsection{Optical/NIR counterparts to the radio emission}

Borys et al. (2004) proposed that the sub-mm emission is related with three objects (see Fig. 1): A LBG (imaged as ARC1 and $\mathrm{ARC} 1 \mathrm{ci})$ and a pair of triply-imaged EROs (B, imaged as $\mathrm{B} 1 / \mathrm{B} 2 / \mathrm{B} 3$; and $\mathrm{C}$, imaged as $\mathrm{C} 1 / \mathrm{C} 2 / \mathrm{C} 3$ ).

As is shown in Fig. 1, ARC1 is situated well inside the region of radio emission associated with $\mathrm{Ra} 1 / \mathrm{Ra} 2$, and is therefore probably related to it. However, in the case of the ERO pair, the images $\mathrm{B} 1$ and $\mathrm{C} 1$ are located at the edge of the radio emission, suggesting that they are not directly contributing a significant amount of the radio flux density in this region.

On the other hand, as shown in Fig. 1, although ARC1ci and TF (an ERO from Takata et al. 2003) are most likely contributing to some of the measured flux density in the "sub-mm toe", B3/C3 is coincident with the maximum of this region. Therefore the EROs are certainly related to the sub-mm emission, so we expect them to be related with the radio emission as well.

The offsets between the estimated centres of the radio emission and the optical/NIR candidates in the image plane are summarised in Table 6. Note that the offsets of ARC1 and the ERO images with respect to Ra1 and Ra2 are larger in declination $\left(\triangle \mathrm{Dec}=3^{\prime \prime}\right)$ than in right ascension $\left(\triangle \mathrm{RA}=2^{\prime \prime}\right)$. This is probably an effect of the magnification produced by the lens cluster, whose largest component is preferentially aligned in the direction of declination (as is reflected in the direction of ARC1 and the overall morphology of the sub-mm and radio emission). In the source plane, our lens model predicts that the offset between $\mathrm{Ra}$ and the ERO pair in the image plane is reduced to $\sim 1^{\prime \prime}$. Indeed, as shown in Sect. 3.2, the LBG and the EROs probably constitute an interacting system, with the radio source Ra situated between them in the source plane. This suggests that the detected radio and sub-mm emission may come from the region in which the systems interact, perhaps due to the enhanced star formation produced by the merging process. A similar phenomena is also observed in the Antennae galaxy, where the bulk of the $\lambda 20 \mathrm{~cm}$ radio emission is situated between the nuclei of both galaxies (see Hummel \& van der Hulst 1986). This scenario can explain the offsets between the radio, sub-mm and NIR emission observed in the region of $\mathrm{ARC} 1, \mathrm{~B} 1$ and $\mathrm{C} 1$ in the image plane.

Moving to the southern region of Fig. 1, the open diamonds correspond to the positions of three additional EROs reported in Takata et al. (2003). Two of these EROs (Tc and Td) are located within 2 arcsec of Rb1 and Rb2 (see Table 6), and may be their NIR counterparts. In this case we expect them to be mirror images, as we assumed in our lens model. We note that Takata et al. argue that these EROs have different photometric redshifts $\left(z_{\mathrm{C}}=3.730, z_{\mathrm{D}}=0.5\right)$, which is inconsistent with that hypothesis. However, both show the same colors (within the errors) in all bands except for B and $I_{\mathrm{c}}$, and in those cases the differences may be due to contamination effects (from the galaxy cluster members situated close to Tc) and the use of a different aperture in each source (see Takata et al. 2003, Table 1). Therefore, with this information we cannot discard the possibility that Tc and Td are lensed images of the same source. On the other hand, the scenario in which Tc and Td are not mirror images (but still the optical/NIR counterparts of $\mathrm{Rb} 1$ and $\mathrm{Rb} 2$ ), is also possible within the lensing context. As is shown in Fig.1, Rb1 is located very close to the critical curve presented in Borys et al. (2004), so a small change in its position can move it right on top of the critical curve (and the source component on top of the caustic), resulting in extremely high magnifications. This is consistent with the non-detection of the predicted counterpart image $\mathrm{Rb} 3$, and the high brightness of Rb1 in the image plane. We also note that the shape of Tc is extremely elongated in the same direction of other faint arcs that appear in the same region of the Hubble image, suggesting that Tc may be lensed.

Another possibility (as discussed earlier in Sect. 4), is that a possible cluster galaxy member could be the NIR counterpart of $\mathrm{Rb} 1$. This scenario can also explain the high brightness of $\mathrm{Rb} 1$ if the galaxy cluster member turns out to be a "radio loud" AGN.

Note that four of the six optical/NIR possible counterparts of the radio/sub-mm emission are EROs, which is consistent with the results presented in Knudsen et al. (2005). On the other hand, studies carried out so far are inconclusive with respect to the overlap between LBGs and SMGs (Chapman et al. 2002b; Adelberger et al. 1998; Webb 2002; Huang et al. 2005). However, in the scenario proposed here, the radio and sub-mm emission is the result of an interaction that involves a LBG, rather than emission coming from the LBG directly.

Apart from the effects of lensing magnification, we also identify four other possible sources of error associated with the measured offset positions. In order of importance these include:

- random measurement errors in the determination of the centre of the unresolved, blended radio components,

- errors in the choice of the position of ARC1, due to its extended and complicated structure, 
Table 6. Suggested NIR counterparts. The columns show: name of the radio source (Radio Source), name of the suggested NIR counterpart (Counterpart Source), coordinates of the counterpart source (RA cs, Dec cs), offsets between the position of the radio source and its NIR counterpart $(\triangle \mathrm{RA}, \Delta \mathrm{Dec})$ and references that contain information about the counterpart sources (References). Note that $\triangle \mathrm{RA}, \Delta \mathrm{Dec}$ should be interpreted as indicative values (see Sect. 4.2 for details). The coordinates are given as offsets with respect to the cluster centre, RA(J2000) $=04: 54: 10.8$ and $\operatorname{Dec}(\mathrm{J} 2000)=-03: 00: 51.6$ (see Takata et al. 2003, Table 2). A version of this table in absolute coordinates can be found in the online material.

\begin{tabular}{clcccll}
\hline \hline Radio Source & Counterpart Source & $\begin{array}{c}\text { RA cs } \\
\left({ }^{\prime \prime}\right)\end{array}$ & $\begin{array}{c}\text { Dec cs } \\
\left({ }^{\prime \prime}\right)\end{array}$ & $\begin{array}{c}\Delta \text { RA } \\
\left({ }^{\prime \prime}\right)\end{array}$ & $\begin{array}{c}\Delta \text { Dec } \\
\left({ }^{\prime \prime}\right)\end{array}$ & References \\
\hline Fb & BCG & 1.1 & -0.8 & 2.4 & 0.0 & Stocke et al. (1999) \\
Fd & 0451-03C & 63.4 & -16.2 & 0.8 & 1 & Stocke et al. (1999) \\
Fe & 0451-03A & -21.6 & -1.2 & 0.7 & 1.1 & Stocke et al. (1999) \\
\hline Ra1 & ARC1 centre & 32.2 & -4.3 & 1.9 & -2.8 & Borys, private comunication \\
Ra1 & ARC1 bottom end & 31.9 & -6.3 & 1.6 & -0.8 & Borys et al. (2004), F720W HST image \\
Ra2 & ARC1 centre & 32.2 & -4.3 & 0.8 & -3.2 & Borys, private comunication \\
Ra2 & ARC1 top end & 32.3 & -1.7 & 0.9 & 2.8 & Borys et al. (2004), F720W HST image \\
Ra2 & B1 & 29.8 & -0.1 & -1.4 & -6.4 & Borys et al. (2004) \\
Ra2 & C1 & 30.0 & 0.1 & -1.4 & -2.9 & Borys et al. (2004) \\
Ra1 & B2 & 28.5 & -9.7 & -1.8 & 2.1 & Borys et al. (2004) \\
Ra1 & C2 & 28.5 & -7.5 & -1.8 & 0.4 & Borys et al. (2004) \\
Rb1 & Tc & 27.9 & -24.7 & 1.4 & -1.3 & Takata et al. (2003) \\
Rb1 & bright galaxy & 24.8 & -25.4 & -1.8 & -0.6 & Borys et al. (2004), F720W HST image \\
Rb2 & Td & 20.5 & -29.7 & -1.8 & -0.8 & Takata et al. (2003) \\
\hline
\end{tabular}

- systematic errors due to offsets between the HST and VLA coordinate reference systems (expected to be up to $\sim 1^{\prime \prime}$, but clearly not dominant since no systematic trend is shown in Table 6).

- intrinsic offsets that can appear if the radio and sub-mm emission come from different regions in the source plane.

Since we cannot properly estimate the contribution of these errors (with the exception of the systematic error), the offsets shown in Table 6 should be taken as indicative values.

\section{Summary and conclusions}

We have presented deep VLA archive observations at $1.4 \mathrm{GHz}$ of the central region of the cluster MS0451.6-0305, discovering multiply-imaged radio counterparts to the sub-mm emission SMM J04542-0301, originally discovered by Chapman et al. (2002a) and recently studied by Borys et al. (2004). This is the second case of multiply-lensed radio emission coming from an intrinsically faint SMG (the first case was SMM J16359+6612 in A2218, see Kneib et al. 2004; Garrett et al. 2005).

With a resolution of $7 \times 6$ arcsec, the radio emission associated with SMM J0452-0301 can best be represented by seven discrete Gaussian components. A simple lens model of this system (based on a NFW mass profile) can reproduce the positions of the radio components assuming that they are multiple images of 3 background sources located at $z=2.9$. However, the model raises some questions that need to be resolved.

Although the radio and sub-mm emission are clearly coincident and present a similar and unusually large angular extent $\left(\sim 1^{\prime}\right)$ and morphology (as expected if the radio and sub-mm emission comes from the same sources), the brightest peak of the radio emission is not coincident with the peak in the sub-mm. We find two possible scenarios that might explain this observation:

- the discrepancy is due to differential magnification produced by the gravitational lensing effect - assuming that the radio and sub-mm emission have differnt morphologies and arise from different regions of the galaxy. Indeed we note, that $\mathrm{Rb} 1$ is situated very close to the critical curve presented in Borys et al. (2004), (Fig. 1).
- the radio emission in that region includes an AGN component associated with an AGN associated with the foreground cluster.

Borys et al. suggested that the sub-mm emission arises from an interacting system of galaxies formed by an ERO pair and a LBG. Although the association of the EROs with the radio emission appears to be uncertain in the image plane (B1 and $\mathrm{C} 1$ are clearly located at the very edge of the radio emission), one of the three radio sources predicted by our lens model ( $\mathrm{Ra}$ ) is situated between the LBG and the ERO pair in the source plane. Our interpretation of this result is that the interacting region of the LBG and the ERO pair might be the source of the radio and sub-mm emission (due to the intense star formation generated during the merging process), whereas the optical/NIR emission might correspond to the cores of the merging galaxies. This scenario (a situation already observed e.g. in the Antennae galaxy) provides a consistent explanation of the offsets between the radio, sub-mm and NIR emission observed in the image plane (upper region of the map).

From the analysis presented in Borys et al. (2004), it is also evident that the LBG and the ERO pair cannot account for all the emission coming from the central region of the sub-mm map. However, the higher resolution VLA observations show extended radio emission located in that region, which is expected to arise from 2 radio sources. Two of the components of that extended emission ( $\mathrm{Rb} 1$ and $\mathrm{Rb} 2)$ seem to be related with another two EROs discovered by Takata et al. (2003) (Tc and Td). Both EROs show similar colors, supporting the idea (assumed in our lens model) that $\mathrm{Rb} 1$ and $\mathrm{Rb} 2$ are images of the same source. However, this latter scenario implies that the photometric redshifts of Tc and Td reported in Takata et al. (2003) may be incorrect. We also found a bright galaxy (probably a cluster member) that can be an optical/NIR counterpart of Rb1, keeping open the possibility that this particular region of the radio map might include emission from a foreground AGN. Unfortunately, none of the evidence is compelling enough to discriminate between the various scenarios that might explain the nature of $\mathrm{Rb} 1$ and $\mathrm{Rb} 2$. 
In summary, we conclude that the radio and sub-mm emission found in MS0451.6-0305 arises from at least 3 highly magnified background sources, one of them being the interacting system proposed by Borys et al. (2004) (an LBG and an ERO pair).

Further progress with this system requires a more complete comparison of multi-wavelength data to be made, and a more detailed lens model to be constructed. Deep, mid-IR observations, as well as higher resolution sub-mm data, might be very important in understanding this system, in particular to confirm the possible lensed nature of $\mathrm{Rb} 1$ and $\mathrm{Rb} 2$. We have recently reobserved MS0451.6-0305 using the VLA at $1.4 \mathrm{GHz}$ in its most extended A-array configuration. These higher resolution observations may shed new light on this system.

Acknowledgements. The authors would like to thank Colin Borys for providing us with the HST/SCUBA reduced images and the contours of the lens model presented in his paper to prepare and analise our figures. We also want to thank James Bullock for providing us his code to estimate the concentration parameter expected for MS0451.6-0305, Charles Keeton for helping to solve problems with the GRAVLENS code, Andy Biggs for his assistance during the data reduction, Antonio Hernán Caballero and Leslie Hunt for providing us with the SED templates, and John Stockes for answering questions related to the bright sources in the field. The authors are also very grateful to the referee for his/her constructive comments which helped to greatly improve the overall manuscript. ABA is also grateful to Edo Loenen for very valuable comments, suggestions and help during the writing of the manuscript. This work was supported by the European Community's Sixth Framework Marie Curie Research Training Network Programme, Contract No. MRTN-CT-2004-505183 "ANGLES".

\section{Appendix A: Calculation of the concentration parameter and the virial mass of the cluster}

The NFW density profile (Navarro et al. 1996) is defined in the three-dimensional space as:

$\rho(r)=\frac{\rho_{\mathrm{s}}}{\left(r / r_{\mathrm{s}}\right)\left(1+r / r_{\mathrm{s}}\right)^{2}}$

where $\rho_{\mathrm{s}}$ is the characteristic density and $r_{\mathrm{s}}$ is the scale radius.

However the GRAVLENS code (Keeton 2001) works with the projected surface mass density of the NFW profile, which is given by:

$\kappa(r)=2 \kappa_{\mathrm{s}} \frac{1-\mathcal{F}}{x^{2}-1} ; \quad \kappa_{\mathrm{s}}=\rho_{\mathrm{s}} r_{\mathrm{s}} / \Sigma_{\text {crit }}$

where $x=r / r_{\mathrm{s}}, \kappa_{\mathrm{s}}$ is the mass scale, and $\mathcal{F}$ is defined as:

$\mathcal{F}(x)= \begin{cases}\frac{1}{\sqrt{x^{2}-1}} \tan ^{-1} \sqrt{x^{2}-1} & (x>1) \\ \frac{1}{\sqrt{1-x^{2}}} \tanh ^{-1} \sqrt{1-x^{2}} & (x<1) \\ 1 & (x=1) .\end{cases}$

Following the formalism used in Bullock et al. (2001), we define the scale radius as:

$r_{\mathrm{s}}=R_{\mathrm{vir}} / c_{\mathrm{vir}}$

where $c_{\text {vir }}$ is the concentration parameter and $R_{\text {vir }}$ is the virial radius.

Comparing the definitions of virial mass used in Navarro et al. (1996) $\left(M_{200}\right)$ and Bullock et al. (2001) $\left(M_{\mathrm{vir}}\right)$, the characteristic density can be written as:

$\rho_{\mathrm{s}}=\rho_{\mathrm{u}}(z) \delta_{\mathrm{c}}$ where $\rho_{\mathrm{u}}(z)$ is the universal density at redshift $z$, and $\delta_{\mathrm{c}}$ is the characteristic over-density, which is linked with $c_{\text {vir }}$ by the following expression:

$\delta_{\mathrm{c}}=\frac{\Delta_{\mathrm{vir}}(z)}{3} f\left(c_{\mathrm{vir}}\right)$

$f\left(c_{\mathrm{vir}}\right)=\frac{c_{\mathrm{vir}}^{3}}{\log \left(1+c_{\mathrm{vir}}\right)-\frac{c_{\mathrm{vir}}}{1+c_{\mathrm{vir}}}}$.

The parameter $\Delta_{\text {vir }}(z)$ is called the virial over-density, and can be approximated (Bryan \& Norman 1998) by:

$\Delta_{\text {vir }}(z) \simeq\left(18 \pi^{2}+82 x-39 x^{2}\right) / \Omega_{\mathrm{m}}(z)-1$

$x=\Omega_{\mathrm{m}}(z)-1$

Combining Eqs. (A.1)-(A.4), the concentration parameter can be calculated using the following expression:

$f\left(c_{\mathrm{vir}}\right)=\frac{3 \kappa_{\mathrm{s}} \Sigma_{\text {crit }}}{\rho_{u}\left(z_{\mathrm{cl}}\right) r_{\mathrm{s}} \Delta_{\mathrm{vir}}\left(z_{\mathrm{cl}}\right)}$

The terms $\kappa_{\mathrm{S}}$ and $r_{\mathrm{s}}$ are given by the lens model (see Table 4). Using the estimated redshift of MS0451.6-0305 from Luppino et al. $(1999)\left(z_{\text {cluster }}=0.55\right), \rho_{\mathrm{u}}\left(z_{\text {cluster }}\right)$ was calculated scaling the value for $z=0$ given in Bullock et al. (2001) $\left(\rho_{\mathrm{u}}(z=0)=\right.$ $8.3 \times 10^{10} h^{2} M_{\odot} \mathrm{Mpc}^{-3}$ ).

Finally, knowing the value of $c_{\text {vir }}$, the virial mass of the cluster can then be estimated as:

$M_{\mathrm{vir}}=\frac{4 \pi}{3} \Delta_{\mathrm{vir}}\left(z_{\mathrm{cl}}\right) \rho_{\mathrm{u}}\left(z_{\mathrm{cl}}\right) R_{\mathrm{vir}}^{3}$.

\section{References}

Adelberger, K. L., Steidel, C. C., Giavalisco, M., et al. 1998, ApJ, 505, 18 Bassino, L. P., Faifer, F. R., Forte, J. C., et al. 2006, A\&A, 451, 789 Blain, A. W. 1997, MNRAS, 290, 553

Borys, C., Chapman, S., Donahue, M., et al. 2004, MNRAS, 352, 759 (B04)

Bryan, G. L., \& Norman, M. L. 1998, ApJ, 495, 80

Bullock, J. S., Kolatt, T. S., Sigad, Y., et al. 2001, MNRAS, 321, 559

Chapman, S. C., Scott, D., Borys, C., \& Fahlman, G. G. 2002a, MNRAS, 330, 92

Chapman, S. C., Shapley, A., Steidel, C., \& Windhorst, R. 2002b, ApJ, 572, L1

Chapman, S. C., Blain, A. W., Ivison, R. J., \& Smail, I. R. 2003, Nature, 422, 695

Comerford, J. M., Meneghetti, M., Bartelmann, M., \& Schirmer, M. 2006, ApJ, 642, 39

Condon, J. J. 1992, ARA\&A, 30, 575

Egami, E., Dole, H., Huang, J.-S., et al. 2004, ApJS, 154, 130

Garrett, M. A. 2002, A\&A, 384, L19

Garrett, M. A., Knudsen, K. K., \& van der Werf, P. P. 2005, A\&A, 431, L21 (G05)

Gioia, I. M., \& Luppino, G. A. 1994, ApJS, 94, 583

Huang, J.-S., Rigopoulou, D., Willner, S. P., et al. 2005, ApJ, 634, 137

Hummel, E., \& van der Hulst, J. M. 1986, A\&A, 155, 151

Hunt, L. K., \& Maiolino, R. 2005, ApJ, 626, L15

Ivison, R. J., Greve, T. R., Smail, I., et al. 2002, MNRAS, 337, 1

Keeton, C. R. 2001, ArXiv Astrophysics e-prints [arXiv: astro-ph/0102340] Kneib, J.-P., Ellis, R. S., Smail, I., Couch, W. J., \& Sharples, R. M. 1996, ApJ, 471,643

Kneib, J.-P., van der Werf, P. P., Kraiberg Knudsen, K., et al. 2004, MNRAS, 349,1211

Knudsen, K. K. 2004, Ph.D. Thesis, Leiden University

Knudsen, K. K., van der Werf, P., Franx, M., et al. 2005, ApJ, 632, L9

Lilly, S. J., Eales, S. A., Gear, W. K. P., et al. 1999, ApJ, 518, 641

Luppino, G. A., Gioia, I. M., Hammer, F., Le Fèvre, O., \& Annis, J. A. 1999, A\&AS, 136, 117 (L99)

Murphy, E. J., Helou, G., Armus, L., et al. 2004, Am. Astron. Soc. Meet. Abstr., 205

Navarro, J. F., Frenk, C. S., \& White, S. D. M. 1996, ApJ, 462, 563

Pointecouteau, E., Arnaud, M., \& Pratt, G. W. 2005, A\&A, 435, 1

Smail, I., Ivison, R. J., \& Blain, A. W. 1997, ApJ, 490, L5

Smail, I., Ivison, R. J., Blain, A. W., \& Kneib, J.-P. 2002, MNRAS, 331, 495

Stocke, J. T., Perlman, E. S., Gioia, I. M., \& Harvanek, M. 1999, AJ, 117, 1967

Swinbank, M., Smail, I., Lindner, C., et al. 2006, ArXiv Astrophysics e-prints

Takata, T., Kashikawa, N., Nakanishi, K., et al. 2003, PASJ, 55, 789 (T03)

Webb, T. M. A. 2002, Ph.D. Thesis 
A. Berciano Alba et al.: Radio counterpart to the sub-mm emission in MS0451.6-0305, Online Material $p 1$

\section{Online Material}


A. Berciano Alba et al.: Radio counterpart to the sub-mm emission in MS0451.6-0305, Online Material p 2

Table A.1. Details of the radio sources observed in the core of MS0451.6-0305. The columns show: absolute coordinates in J2000 (RA, Dec), peak flux density $\left(S_{\mathrm{Pk}}\right)$, total flux density $\left(S_{\mathrm{T}}\right)$ and deconvolved Gaussian sizes (major axis, minor axis and position angle) with their corresponding formal errors.

\begin{tabular}{lccccccc}
\hline \hline Name & $\begin{array}{c}\text { RA }\left(+4^{\mathrm{h}} 54^{\mathrm{m}}\right) \\
\mathrm{J} 2000(\mathrm{~s})\end{array}$ & $\begin{array}{c}\text { Dec }\left(-3^{\circ}\right) \\
\mathrm{J} 2000\left({ }^{\prime},{ }^{\prime \prime}\right)\end{array}$ & $\begin{array}{c}S_{\mathrm{Pk}} \\
\mu \mathrm{Jy}\end{array}$ & $\begin{array}{c}S_{\mathrm{T}} \\
\mu \mathrm{Jy}\end{array}$ & $\begin{array}{c}\text { Maj Axis } \\
{ }^{\prime}\end{array}$ & $\begin{array}{c}\text { Min Axis } \\
{ }^{\prime \prime}\end{array}$ & $\begin{array}{c}\text { PA } \\
\text { deg }\end{array}$ \\
\hline $\mathrm{Ra} 2$ & $12.89 \pm 0.02$ & $00,50.49 \pm 0.43$ & $70 \pm 8$ & $95 \pm 18$ & $6 \pm 1$ & $2 \pm 1$ & $27 \pm 28$ \\
$\mathrm{Ra} 1$ & $12.82 \pm 0.01$ & $00,58.66 \pm 0.22$ & $109 \pm 9$ & $109 \pm 9$ & - & - & - \\
$\mathrm{Rb} 1$ & $12.57 \pm 0.01$ & $01,17.58 \pm 0.16$ & $151 \pm 9$ & $151 \pm 9$ & - & - & - \\
$\mathrm{Rb} 2$ & $12.29 \pm 0.03$ & $01,22.10 \pm 0.71$ & $52 \pm 8$ & $100 \pm 22$ & $9 \pm 2$ & $3 \pm 2$ & $158 \pm 14$ \\
$\mathrm{Rc} 1$ & $12.27 \pm 0.04$ & $01,09.86 \pm 0.43$ & $50 \pm 9$ & $55 \pm 16$ & $6 \pm 2$ & - & $112 \pm 11$ \\
$\mathrm{Rc} 2$ & $11.70 \pm 0.04$ & $01,22.93 \pm 0.81$ & $41 \pm 8$ & $58 \pm 18$ & $7 \pm 2$ & $1 \pm 3$ & $10 \pm 163$ \\
$\mathrm{Rc} 3$ & $13.16 \pm 0.05$ & $00,40.69 \pm 0.46$ & $52 \pm 8$ & $78 \pm 19$ & $8 \pm 2$ & $0 \pm 2$ & $73 \pm 11$ \\
\hline $\mathrm{Fa}$ & $11.47 \pm 0.04$ & $01,04.34 \pm 0.57$ & $45 \pm 9$ & $50 \pm 17$ & $4 \pm 6$ & $0 \pm 4$ & $123 \pm 38$ \\
$\mathrm{Fb}$ & $11.03 \pm 0.04$ & $00,52.35 \pm 0.59$ & $49 \pm 9$ & $70 \pm 20$ & $6 \pm 5$ & $3 \pm 6$ & $122 \pm 45$ \\
$\mathrm{Fc}$ & $14.16 \pm 0.04$ & $01,29.06 \pm 0.55$ & $44 \pm 9$ & $44 \pm 9$ & - & - & - \\
$\mathrm{Fd}$ & $15.08 \pm 0.003$ & $01,08.78 \pm 0.05$ & $634 \pm 9$ & $1039 \pm 21$ & $7.61 \pm 0.13$ & $1.36 \pm 0.45$ & $128 \pm 1$ \\
$\mathrm{Fe}$ & $09.31 \pm 0.001$ & $00,51.53 \pm 0.02$ & $1549 \pm 9$ & $1777 \pm 17$ & $3.3 \pm 0.1$ & $0.8 \pm 0.5$ & $125 \pm 3$ \\
\hline
\end{tabular}

Table A.2. Suggested NIR counterpart sources of some radio emissions. The columns show: name of the radio source (Radio Source), name of the suggested NIR counterpart (Counterpart Source), absolute coordinates of the counterpart source in J2000 (RA cs, Dec cs), Radio-NIR offsets $(\triangle \mathrm{RA}, \Delta \mathrm{Dec})$ and references that contain information about the counterpart sources (References).

\begin{tabular}{|c|c|c|c|c|c|c|}
\hline Radio Source & Counterpart Source & $\begin{array}{c}\mathrm{RA} \operatorname{cs}\left(+4^{\mathrm{h}} 54^{\mathrm{m}}\right) \\
\left({ }^{\prime \prime}\right)\end{array}$ & $\begin{array}{c}\operatorname{Dec} \operatorname{cs}\left(-3^{\circ}\right) \\
\left({ }^{\prime \prime}\right)\end{array}$ & $\begin{array}{c}\Delta \mathrm{RA} \\
\left({ }^{\prime \prime}\right)\end{array}$ & $\begin{array}{c}\Delta \mathrm{Dec} \\
\left({ }^{\prime \prime}\right)\end{array}$ & References \\
\hline $\mathrm{Fb}$ & BCG & 10.73 & $00,50.81$ & 2.39 & 0.04 & Stocke et al. (1999) \\
\hline $\mathrm{Fd}$ & $0451-03 \mathrm{C}$ & 15.04 & $01,08.11$ & 0.75 & 0.97 & Stocke et al. (1999) \\
\hline $\mathrm{Fe}$ & 0451-03A & 9.08 & $00,52.69$ & 0.73 & 1.143 & Stocke et al. (1999) \\
\hline Ral & ARC1 centre & 12.946 & $00,55.900$ & 0.83 & 1.9 & Borys, private comunication \\
\hline Ra1 & ARC1 bottom end & 12.926 & $00,57.900$ & 1.62 & -0.759 & Borys et al. (2004) ${ }^{a}$ \\
\hline $\mathrm{Ra} 2$ & $\mathrm{ARC} 1$ centre & 12.946 & $00,55.900$ & -3.19 & 2.76 & \\
\hline $\mathrm{Ra} 2$ & ARC1 top end & 12.953 & $00,53.280$ & 0.94 & 2.79 & Borys et al. (2004) ${ }^{a}$ \\
\hline $\mathrm{Rb} 1$ & $\mathrm{Tc}$ & 12.66 & $01,16.3$ & 0.54 & 1.28 & Takata et al. (2003) \\
\hline $\mathrm{Rb} 1$ & galaxy & 12.453 & $01,16.98$ & -1.75 & -0.60 & Borys et al. (2004) ${ }^{a}$ \\
\hline $\mathrm{Rb} 2$ & $\mathrm{Td}$ & 12.17 & $01,21.3$ & 1.79 & 0.80 & Takata, private communication \\
\hline
\end{tabular}

From F720W HST image. 